\title{
HUMAN EARPRINTS: A REVIEW
}

Nitin Kaushal ${ }^{1 *}$ and Purnima Kaushal ${ }^{2}$

${ }^{1}$ Department of Oral Pathology, B.R.S. Dental College and Hospital, Village Sultanpur, Panchkula, Haryana, INDIA

${ }^{2}$ Department of Anthropology, Punjab University, Chandgarh, INDIA

\begin{abstract}
Since centuries the external ear which is known as the pinna or the auricle has been used as a means of identification. It has been studied and described as a part of procedures to establish the identity of criminals and victims of crimes and accidents. Not only the auricle itself showed potential for establishing the identity of criminals, but also its prints. When perpetrators of crimes listen at, for instance, a door or window before breaking and entering, oils and waxes leave prints that can be made visible using techniques similar to those used when lifting fingerprints. These prints appear characteristic for the ears that made them.
\end{abstract}

Keywords: Earprints; Forensic; Biometrics

\section{Introduction}

Try a simple experiment; try to visualize what your ears look like. You were not able to? Well, then try to describe the ears of someone you see every day. You will find that even if you are looking directly at someone's ears, they are still difficult to describe. On the other hand, we are all capable of describing the faces of even briefly glimpsed strangers with significant detail to allow police artists to reconstruct remarkable resemblances of them [1]. In 1854 Armede Joux wrote "Show me your ear and I'll tell you who you are, where you came from and where you are going". Joux published these words in "Gazette des hopitaux de Paris 1854". Of course the author was not able to produce a portrait of neither a person, nor his present address or where he was heading to, just like the knowledge of the color of eyes or hair of a person does not yet provide a portrait of the individual. If we look further in the history of ears and ear prints we will find Darwin, who attracted the attention of the scientific world regarding the ear, during his research about the relation with primates, by saying that the ear is one of the elementary organs. To prove for his position he pointed at the broadening of the middle of the helix (auricular tubercle), indicating that this is nothing else but a corner of the primitive ear which is reducing. Science recognized this reducing of the corner and has tributed this part by naming it "tubercle of Darwin" [2].

In order to recognize the various components that make out an earprint, one first needs to familiarize oneself with the anatomy of auricle [3]. An auricle is an irregular, oblong dermal plica, which embraces the orifice of external auditory meatus. The auricle is almost twice as long as its width and its size vary significantly. The auricle is bent in many directions. Its structure is supported by elastic cartilage. The cartilage skeleton determines most of auricle shape. Only the lower ear-lobe is deprived of cartilage. The auricle belongs to the organs characterized by location, size and shape, which are individual features for each human being. Numerous studies carried out in the world have demonstrated that the shape of auricle generally does not change throughout human life and this organ is not very much prone to injury. Thus one can talk about biological features of auricle similarly to biological features of fingertip skin ridges pattern, attributing such notions as individuality, unchangingness (stability) and indestructibility. The skin of the auricle is thoroughly covered with sebaceous substance that comes from sebaceous glands and is transferred from the hair. When a human ear is pressed to a surface, oils and waxes leave behind a two dimensional representation known as an ear print [4].

The human auricle has numerous applications in forensic science.
An ear constitutes a valuable identification appearance feature used in creating a signalment portrait, various methods of appearance reconstruction, identification of persons based on photographs and identification of corpses. Another important aspect is identification of ear impressions on various surfaces found at the scene of crime [5]. The ear lobe is actually a part of the disaster identification system [6].

\section{Structure of human ear}

Ear has a definite structure just like the face. As shown in (Figure 1) the shape of the ear tends to be dominated by the outer rim or helix, and also by the shape of the lobe. There is also an inner helix or antihelix which runs roughly parallel to the outer helix but forks into two branches at the upper extremity. The inner helix and the lower of these two branches forms the top and left side of the concha, named for its shell-like appearance. The bottom of the concha merges into the very distinctive intertragic notch. Note also the crus of helix where the helix intersects with the lower branch of the antihelix. This is one of the points used by Iannarelli as a reference point for his measurement system, the other point being the antitragus or the little bump on the left of the intertragic notch. The front of the concha opens into the external ear canal or acoustic or auditory meatus. Some ears have well formed lobes, whereas others have almost none [7]. Characteristic features of ear prints towards individualizing properties as shown in (Figure 2) are as follows: Starting with the crus of helix and then follow the outer rim clockwise: The crus of helix has a variety of shapes and is one of the features that will almost always leave a print when an ear is pressed against a surface. The helix rim is responsible for the shape of the ear. The shape of the rim itself can be very diverse. In cross section it can be completely rolled as well as unrolled. The place where the unrolling starts or ends is different for everybody. An important role in the identification process is the inside edge of the helix rim. It may contain either notches or knobs and can have clearly visible angles. At around two o'clock there is the auricular tubercle or Knob of Darwin.

${ }^{*}$ Corresponding author: Nitin Kaushal, Department of Oral Pathology, B.R.S Dental College and Hospital, Village Sultanpur, Panchkula, Haryana, India, Tel: 09780671752; E-mail: nitinzkaushal@yahoo.co.in

Received June 26, 2011; Accepted November 21, 2011; Published December 25, 2011

Citation: Kaushal N, Kaushal P (2011) HUMAN EARPRINTS: A REVIEW. J Biomet Biostat 2:129. doi:10.4172/2155-6180.1000129

Copyright: ( 2011 Kaushal N, et al. This is an open-access article distributed under the terms of the Creative Commons Attribution License, which permits unrestricted use, distribution, and reproduction in any medium, provided the original author and source are credited. 


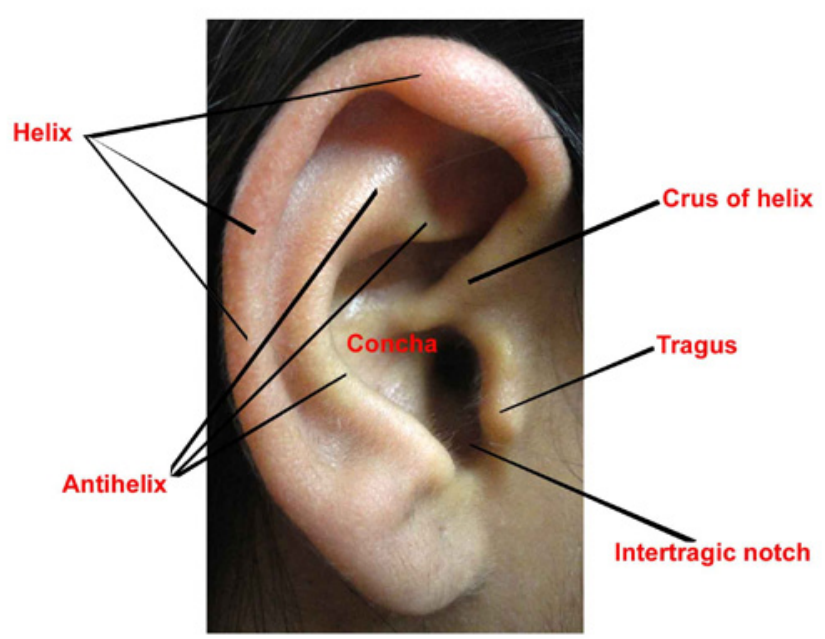

Figure 1: Anatomical landmarks of Human Ear.

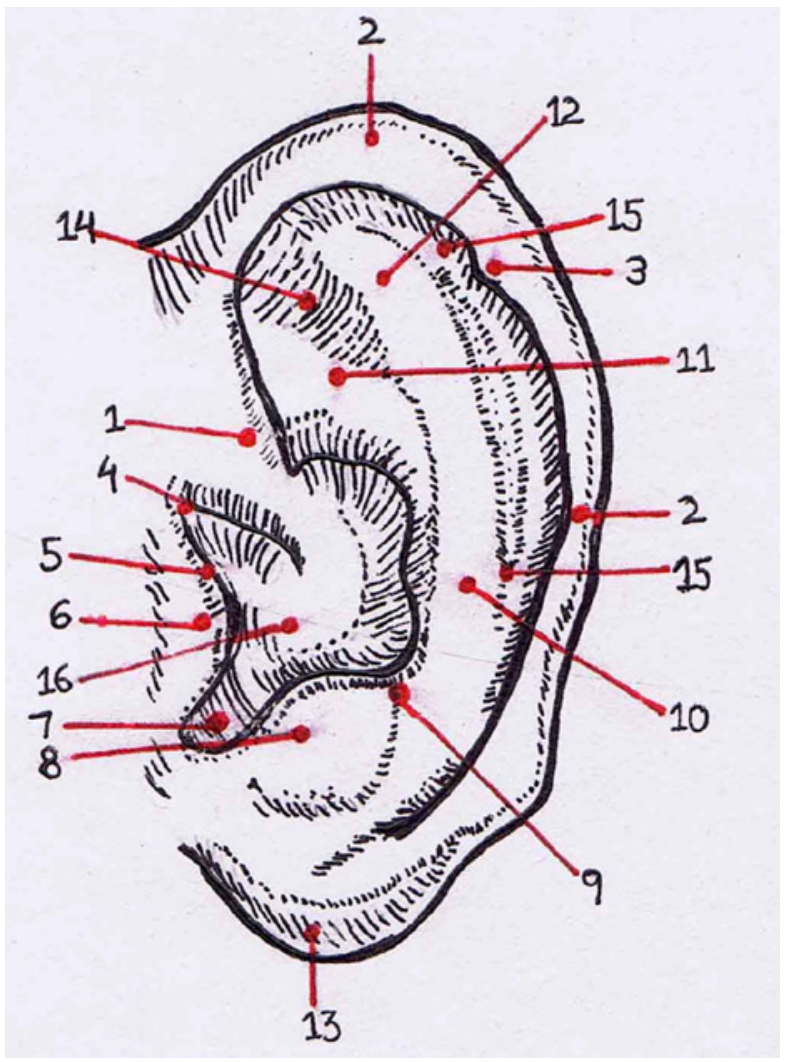

Figure 2: Sketch of Human ear showing various anatomical landmarks.

This feature is not present in all ears. In one person it can occur in one ear only, so either left or right. Also multiple knobs are possible. They can be situated in the inside of the rim, on the outside, on both sides or only on the rim itself.

Starting again at the crus of helix and now going counter clock-wise there are features like the anterior notch and the anterior knob. These features are not present in all ears. Sometimes they can be observed in the ear itself but not be visible in the ear print because of pressure.
Tragus: The tragus is in fact a protection "lid" of the auditory canal. When the head is pressed to a surface very hard, it will close the opening. Intertragic notch: The intertragic notch lies in between the tragus and anti-tragus. Its shape depends on the shape and size of these features. It can be round, horse-shoe shaped of v-shaped. Anti-tragus: The anti-tragus can be dominant till hardly noticeable. Posterior auricular furrow: This feature is a groove or furrow between the anti-tragus and the anthelix and is not present in all ears. Ant-helix: Lower- and upper crus of ant helix. The ant-helix comes in many shapes and can, together with lower and upper crus be divided into different classes. At the bottom we have the lobule or earlobe, which can have various shapes such as triangular, round, rectangular and lobbed [2]. According to the shape of the earlobe, the ear may be classified as kidney-shaped i.e. oval with an unattached earlobe or shaped as half of a heart i.e. oval with attached earlobe [3].

The overall shape of the auricle is determined by the contour of the helix and of the lobule. A common way of classifying the shape of the auricle is by defining it as either oval, round, rectangular and triangular. According to the definitions provided by Van der Lugt the oval ear is longer than it is wide, the width being maximal at the centre. It has a rounded top and bottom. The round ear is almost as long as it is wide. It also has a rounded top and bottom. The rectangular ear is longer than it is wide, with a rectangular top and bottom. Both ends are almost as wide as halfway down the ear. The triangular ear is longer than it is wide, with a rounded top that is wider than the bottom [3] Length (or height) and width of the ear may be determined according to various standards. According to the method used by Meijerman et al., the distance between the most superior point of helix and the most inferior point of the earlobe on lines parallel to the ear base (the auricular attachment to the head) determines the length of the auricle. Width of the auricle is determined by the greatest distance between the ear base and the posterior part of the helix, on a line perpendicular to the ear base. Sexual dimorphism is evident in measurements of length and width of the auricle. Among males and females belonging to the same age group, males exhibit higher values for both auricle length and width [3].

The majority of crime scenes where earprints are discovered involve burglaries [3]. At the scene of crime, offenders often leave ear prints on the windows and doors. A latent earprint is the product of the secretions (fats and waxes) of the ear coming into contact with a surface. Hormones regulate these secretions and the amount of fats and waxes on the surface of the ear can vary from individual to individual. In cases where the secretions are present in large quantities a very clear latent earprint can be produced (8). An individual intent on burglary may press his ear against a window or door in order to ascertain whether or not the location is occupied [4]. Investigations into earprint identification found differences between certain eaprints of one donor. The prints that displayed these differences were 'listening' earprints i.e. those produced by the donor when carrying out the act of listening against a surface, and a 'non-listening' earprints i.e. those produced by the donor simply pressing the ear against a surface but not carrying out the act of listening. It was found that for a particular individual, when the function of listening was carried out the physiological changes of the outer ear were different to those changes brought on by just simply applying pressure to the ear. The anti-helix popped out and produced a type of double print when the donor tried to listen against a surface, but by just applying pressure to the ear the anti-helix did not react in the same way. Another difference that was found between the two prints was that the print produced by the individual carrying out the act of 
listening was angled; the inferior section of the lobule was pointing toward the seven o'clock position. Whereas on the non-listening earprint the same section was directed almost vertically downwards [8].

Alternatively a person planning to commit a murder or rape will leave ear print when trying to locate the position of his or her future victim. If such a crime is committed, the police will begin an investigation, and scene of crime officers will process the crime scene. They can then recover the earprints. This ear print will contain information about offender's ears, representing its anatomical features. The ear print can later be compared to other crime scene prints and to ear prints taken under controlled conditions from suspects [4].

For recovery purposes fingerprint powders are used and the mark is lifted on foil. Comparative material in case of auricle mark identification needs to be quite extensive. Reference impressions should be made with different pressure and at various angles. Comparative impressions should be taken with a pressure force of $1 \mathrm{~kg}, 2 \mathrm{~kg}, 3 \mathrm{~kg}$, which is feasible with use of a U-1 kit. The U-1 kit is designed for collecting comparative material with controlled pressure. The kit consists of scales and a set of gripping devices. An impression can be collected directly on black celluloid or glass plate. The comparative impression is covered a aluminium fingerprinting powder and fixed 6 .

In the Central Forensic Laboratory of Polish Police (Warsaw) identification of auricle traces is carried out in the following stages:

1. Assessment of evidential and comparative material involves checking whether evidential trace contains sufficient number of characteristics enabling identification. Subsequently the comparative material is checked with respect to quality and legibility. It has to be decided whether material is sufficient for examination and, if necessary, it can be complemented with additional impressions.

2. Group identification examination involves comparing size and topography of auricle in evidential trace and comparative traces.

3. The contour method involves drawing the contour of the auricle on transparent foil and comparing the contour with the comparative print. This technique allows precise determination of distances and relative location of individual characteristics.

4. The method of determining common features involves comparison of such auricle parts as helix, anti helix and anti helix region as well as concha, tragus, anti tragus and lobule. In those parts, 24 areas are differentiated. The analysis of anatomy, type and distribution of those areas justify their status of individual characteristics.

Basing on statistical calculations, demonstrating conformity of 7 characteristics in evidential and comparative impression has been decided as sufficient to conclude that they come from the same person [5].

In another method, FearID listening box is used. This apparatus had a weight scale incorporated in one of its surfaces. The surface of the weight scale served as the listening surface, enabling the measurement of (perpendicular) force applied by the ear during listening. Loudspeakers were placed on the inside of sound box, making it possible to supply sound during a listening effort. After the sound box had been adjusted for the appropriate height, the subject was asked to approach the sound box and to listen for sound. Latent prints were enhanced with special silver fingerprint powder referred to as aluminium powder using a brush made of squirrel hair. Prints were secured using black gelatine lifters. These lifters comprised of three separate layers: a removable transparent plastic cover over a layer of low tack gelatine with a backing of white rubberised linen. Firstly, the transparent top layer was removed, after which the gelatine layer was applied onto the powdered earprint. The lifter now having the fingerprint powder adhered to it was removed from the listening surface and the transparent top layer was placed back on the gel to protect the print [3].

Potentially ear-print evidence is as powerful as other types of body trace evidence, such as that provided by finger prints and DNA. In addition, though criminals have learned to tamper with fingerprints and DNA evidence, ear-print evidence is more resistant to such attack. Like fingerprints and DNA, ear-print evidence can be used to place a suspect at the scene of a crime. The value of this is exemplified by the fact that there are sometimes complications with fingerprint and DNA evidence. For example, crime scenes are often contaminated by the fingerprints and/or DNA of people unrelated to the offender, such as relatives or friends of the victim. Furthermore, it is possible for the offender to implicate an innocent individual by placing genetic material and/or fabricated fingerprints at the crime scene. However, these complications are less likely for ear-prints, since a subject's cooperation is required to obtain an ear-print. An additional benefit of ear-prints is that they can be used to corroborate fingerprint and DNA evidence [4].

Ears can be used in identification of unknown persons especially in cases of mass disasters, burns, drowning etc. where the face is severely disfigured [9]. The identity can be established through methods based on the morphology and measurements of ears of the victim 13. Post mortem photographs of left and right ears taken are compared with the victim's ante mortem photographs supplied by his family [9]. Among the various parts of the pinna, the ear lobe is more often used in forensic cases. The shape of the lobe can vary from well formed to attached [6]. Whether the ear lobe is attached or not is an international standard for identification in disaster victim identification 8.In India, morphology of ear was used to confirm the identity of Veerappan, the sandal wood smuggler who was killed by Special Task Force in 200413. Ear piercing, which often occurs on the lobe, is also a useful attribute for forensic identification [6].

Ear as a biometric: Both terms biometrics and biometry have been used since early in 20th century to refer to the field of development of statistical and mathematical methods applicable to data analysis problems in biologic sciences14. Recently the term biometrics has been used to refer to the emerging field of technology devoted to identification of individuals on the basis of their biological traits [10]. A biometric is any human feature that can be measured and used for automated or semi automated identification. Biometrics is a method of identifying or verifying the identity of an individual based on the physiological and behavioral characteristics [9]. Among all the biometric techniques, fingerprint based identification is the oldest method which has been successfully used in numerous applications [10]. Other common examples are iris pattern and facial patterns; less well known are the ear, body odour and gate [11]. Biometrics can be either passive or active. Facial recognition, for example is a passive biometrics. It does not require user's active participation and can be successful without persons even knowing that they have been analysed [9]. Ears have also been proposed as a new class of biometrics for passive identification which have both reliable and robust features which are extractable 
from a distance [1]. Active biometrics such as fingerprint, retina scanning, signature recognition etc. however, do require personal cooperation and will not work if one denies participation in the process [9]. Another type of biometrics is the automated biometrics which can be either physiological (e.g., ear, face, hand, eye, finger print) which are based upon measurements of external physical traits, or behavioral (e.g., signature, voice, and keystroke) which usually measure learned behaviors which are dependent upon environmental factors [12]. There are different methods to verify identity and the most commonly used methods are:

\section{(i) Possessions, like cards, badges, keys}

(ii) Knowledge, like userid, password, Personal Identification Number

(iii) Biometrics like ear, fingerprint, face

Possessions can be lost, forgot or replicated easily. Knowledge can be forgotten. Both possessions and knowledge can be stolen or shared with other people. However in biometrics these drawbacks do exist only in small scale [11].

Amongst the numerous methods of ear identification, the common ones are: (i) taking a photo of an ear, (ii) taking "earmarks" by pushing ear against a flat glass and (iii) taking thermogram pictures of the ear [11]. Alfred Iannerelli made two large scale ear identification studies. The first study compared 10,000 ears drawn from a randomly selected sample in California and the second study examined identical twins and triplets (who have identical genetic makeup). In both the studies all examined ears were found to be unique, though identical multiple birth siblings had similar, but not identical ear structures [9]. An anthropometric technique was developed by A. Iannerrilli in 1949. It is based on the 12 measurements as shown in (Figure 2). The locations shown are measured from specially aligned and size-normalized photographs. Each photograph is aligned during development so that the lower tip of a standardized vertical guide on the development easel touches the upper flesh line of the Concha area while the upper tip touches the outline of the Antitragus. Since each ear is aligned and scaled during development, the resulting photographs are normalized in size and orientation, enabling the extraction of comparable measurements directly from the photographs. The distance between each of the numbered areas in figure is measured in units of $3 \mathrm{~mm}$ and assigned an integer distance value. These twelve measurements, along with information on sex, race, are then used for identification [12].

Inspired by the work of Iannarelli, Burge and Burger conducted a proof of concept study where the viability of ear as a biometric was shown both theoretically in terms of uniqueness and measurability over time and in practice through the implementation of a computer vision based system. Each subject's ear was modeled as an adjacency graph built from the Voronoi diagram of its Canny extracted curve segments. They devised a novel graph matching algorithm for authentication which takes into account the erroneous curve segments which can occur in the ear image due to changes such as lighting, shadowing and occlusion [7].

Principal component analysis (PCA) is by far the most widely adopted methods used in ear biometrics. PCA is a technique for reducing the dimension of feature vectors while preserving the variation in the dataset. A low dimension space called 'Eigen space', which is defined by a set of 'Eigen vectors' of dataset is used in classification [9]. Chang et al. compared the performances of PCA when applied on face and ear recognition. It was found that there was no significant difference between the face and ear in terms of recognition performance [9]. Hurley et al. developed an invertible transform which transforms an ear image into a force field by pretending that pixels have a mutual attraction proportional to their intensities and inversely to the square of the distance between them rather like Newton's Universal Law of gravitation [7]. Underlying this force field there is an associated energy field which in the case of ear takes the form of a smooth surface with a number of peaks joined by ridges [7]. The peaks correspond to potential energy wells and to extend the analogy the ridges correspond to potential energy channels [7]. Since the transform also turns out to be invertible, all of the original information is preserved and since the otherwise smooth surface is modulated by these peaks and ridges, it is argued that much of the information is transferred to these features and that therefore they should make good features [7].

The ear may also be pressed against some material, e.g. glass, and the earmark can be used as a biometric [11]. The main problem with using ear biometrics is that they are not usable if the ear is covered e.g. with a hat or hair. In active identification systems the subject can take the hat off or pull their hair back for authentication. The main problem occurs in passive identification systems [11]. In the case of the ear being only partially occluded by har, it is possible to recognize the hair and segment it out of the image [1]. This can be done by using texture and color segmentation or by using thermogram images. A thermogram image is the one in which the surface heat (i.e., infrared light) of the subject is used to form an image [1]. In the thermogram picture's different colors and textures are used to find different parts of ear [11] Hair being at a lower temperature than ear, especially external auditory meatus, can be segmented out from the image [9]. The meatus of the ear is easily localized using thermogram imagery [1]. In a profile image of a subject, if the ear is visible, then the meatus will be the hottest part of the image, with an expected eight degree Celsius temperature differential between it and the surrounding hair [1].

\section{Conclusion}

Earprints are found at various crime scenes and the majority of the scenes of crime where earprints are discovered involve burglaries. In the contemporary forensic science more and more attention has been paid to those traces, which until recently were considered untypical, such as ear impressions [5]. However the ear print evidence has not been exploited as widely as other forms of body trace evidence [4]. The extraction of biometric data from crime scene traces is an integral part of the forensic process [13]. Studies have shown that biometrics based upon ear is viable in that the ear anatomy is probably unique to each individual and that the features based upon measurements of that anatomy are comparable over time. Given that they are viable, identification by ear biometrics is promising because it is passive like face recognition, but instead of the difficult to extract face biometrics it can use robust and simply extracted biometrics like those in fingerprints [12]. One may add that though ear is still an infant in the ever enlarging field of biometrics, it is already proving its grit and is on the verge of emerging as a major biometric tool [9].

\section{References}

1. Burge M, Burger W (2002) Ear Biometrics. In Jain A, Bolle R \& Pankanti S (Eds). Biometrics: Personal identification in networked society, Kluwer Academic, Dordrecht, 273-286.

2. Van der LC (2001) Ears and earprints, individualizing crime scene marks Problems of Forensic Sciences 56: 38-45.

3. Meijerman L (2006) Inter and intra individual variation in earprints. PhD Thesis Leiden University. 
4. Kennerley J (2000) Ear prints in criminal investigation. The European Commission Community Research.

5. Kasprazak J (2001) Identification of ear impressions in polish forensic practice. Problems of Forensic Sciences 57: 168-174

6. Nixon MS, Bouchrika I, Arbab-Zavar B, Carter JN (2010) On use of biometrics in forensics: Gait and ear. Proceedings of the 18th European Signal Processing Conference, August 23-27, Aalborg, Denmark.

7. Hurley DJ, Arbab-Zavar B, Nixon MS (2007) The ear as a biometric Proceedings of the 15th European Signal Processing Conference, September 3-7, Poznan, Poland.

8. Johnson Z. Standard operating procedures for colleting the earprint validation sample for the 'FEARID' forensic ear identification project. United Kingdon: NTC Aykely Heads Durham.
9. Purkait R (2007) Ear Biometric: An aid to personal identification. Anthropologist 3: $215-218$.

10. Bohm I, Testor F (2007) Biometric systems.

11. El-Bakry HM, Mastorakis N (2009) Ear recognition by using neural networks. Proceedings of the 11th International Conference on Mathematical methods and computational techniques in Electrical engineering; September 28-30; Athens, Greece.

12. Burge M, Burger W (1998) Using ear biometrics for passive identification Chapman and Hall, New York, USA.

13. Lopatka M (2008) Learning appropriate ear-print features for use as biometric traces. 